\title{
Isokinetic dynamometer evaluation of the effects of early thigh diameter difference on thigh muscle strength in patients undergoing anterior cruciate ligament reconstruction with hamstring tendon graft
}

\author{
Bekir Eray Kılınç ${ }^{1, *}$, Adnan Kara², Savas Camur ${ }^{3}$, Yunus $0 c^{4}$, Haluk Celik ${ }^{4}$ \\ ${ }^{1}$ Igdir State Hospital Orthopaedics and Traumatology Department, Igdir, Turkey \\ ${ }^{2}$ Istanbul Medipol University Orthopaedics and Traumatology Department, Istanbuk, Turkey \\ ${ }^{3}$ Agri Dogubayazıl State Hospital Orthopaedics and Traumatology Department, Agri, Turkey \\ ${ }^{4}$ Sisli Etfal Training and Research Hospital Orthopaedics and Traumatology Department, Istanbul, Turkey
}

After anterior cruciate ligament (ACL) reconstruction, which muscle groups are more affected from frequently developing thigh muscle atrophy is a matter of debate. We evaluate the effect of thigh circumference difference between patients' knees who were administered the ACL reconstruction with hamstring tendon autograft and intact knees, on torque between the hamstring and quadriceps muscles. Fifty-five patients at least 6 months follow-up period available were included in our study. Power measurements of quadriceps and hamstring muscle groups in patients' extremities were done by using isokinetic dynamometer. The maximum torque values at $60 \% \mathrm{sec}, 240 \% \mathrm{sec}$ in frequency, positions of flexion and extension were determined. In accordance with our findings it is still possible to encounter the thigh atrophy in average 28 months after ACL reconstruction surgery even under physical rehabilitation programs and appropriate follow-up. It is inevitable for the clinician to consider these changes in diagnosis and rehabilitation stages. It can't be ignored that muscle weakness mechanisms developing in the thigh circumference vary according to the thigh muscle group and knee flexors play an important role in thigh atrophy when determining an appropriate rehabilitation program after reconstruction application.

Keywords: Thigh diameter, Muscle torque, Cruciate ligament repair

\section{INTRODUCTION}

Anterior cruciate ligament (ACL) has a primary role in the limitation of anterior translation of the tibia as regards to the femur. Hamstrings play a major role in protecting the ACL. They are also important for the compensation of stability in the ACL in the knee lesions and patients with ACL. The hamstring muscle weakness and hamstrings' weak relationship with the quadriceps are risk factors for ACL injuries. Various surgical methods are defined for in the treatment of anterior cruciate ligament. One of the most commonly used treatments is the method in which knee flexor tendons are used as autograft. As shown in previous studies, after using this method reconstructed knee recovered up to the $90 \%$ of flexion muscle strength of counter knee (Harter et al.,1990; Lipscomb et al.,1982; Simonian et al.,1997). It is also shown in previous studies that semitendinosus which is also used as ACL graft has regeneration potential from more proximal and with similar morphology (Eriksson et al., 2001; Ferretti et al., 2002; Hioki et al., 2003; Nakamae et al., 2005; Simonian et al., 1997; Williams et al., 2004). Although there is a development of regeneration close to normal morphology; knee flexors' abnormalities in neurological function can be a barrier to motor units' function by preventing their healing. Hamstring weakness after ACL rupture is associated with weakness in knee function. However, morphologi-
${ }^{*}$ Corresponding author: Bekir Eray Kilınç

Igdir State Hospital Orthopaedics and Traumatology Department, Devlet Hastanesi Kışla Mahallesi 76000 Iğdır, Turkey

Tel: +90-530-606-1884, Fax: +90-530-606-1884, E-mail: dreraykilinc@gmail.com

Received: February 12, 2015 / Accepted: April 8, 2015
This is an Open Access article distributed under the terms of the Creative Commons Attribution Non-Commercial License (http://creativecommons.org/licenses/by-nc/3.0/) which permits unrestricted non-commercial use, distribution, and reproduction in any medium, provided the original work is properly cited. 
cal factors such as muscle atrophy in these patients is not the only factor that determines the maximum power; in previous studies, it is shown that despite the absence of morphological abnormalities the afferent feedback deficiency from ACL may interfere with the activation of gamma motor unit of the muscles around the knee (Konishi et al., 2002a; 2002b; 2003; 2007a; 2007b).

Therefore, even if the knee flexor tendon regeneration is morphologically normal and that motor unit complex may prevent the function of the knee flexors by causing neurologic abnormalities. Therefore, while comparing knee flexor muscle strength of patients who underwent ACL reconstruction with normal uninjured knee, muscle weakness due to the possibility of neurologic abnormalities can not be ignored.

After ACL reconstruction, which muscle groups are more affected from frequently developing thigh muscle atrophy is a matter of debate. Muscle strength tests are used to evaluate recovery of ACL after treatment and the effectiveness of treatment. According to the study carried out, after ACL reconstruction using hamstring tendon grafts, weakness in the knee flexor muscle strength can be observed up to 24 months. In addition, previous studies showed that in patients who underwent ACL reconstruction quadriceps muscle torque per unit volume is significantly lower compared the intact knee (Konishi et al., 2007b).

In our study, we aimed to evaluate the effect of thigh circumference difference between patients' knees who were administered the ACL reconstruction with hamstring tendon autograft and intact knees, on torque between the hamstring and quadriceps muscles. As is known, development of muscle weakness in quadriceps after ACL lesions caused by neurological dysfunction was shown to be a natural consequence. In our study, we planned to evaluate the effect of thigh circumference difference between operated extremity and intact extremity on knee flexors'muscle strength.

\section{MATERIALS AND METHODS}

Fifty-five patients ( 54 males, 1 female, mean age $28.15 \pm 6.47$ ) who underwent ACL reconstruction in our clinic and with at least 6 months follow-up period available were included in our study. While choosing the patients, not having any disease nor interventions previously on intact knees has taken into account. Patients who have symptoms and signs such as discharge, inflammation, instability, locking, limitation of motion, anxiety while exercising were removed from the study. All operations were applied in teaching and research hospital by the same surgeon. The condition that autogenous hamstring tendon graft usage in ACL recon- struction was searched for the patients. In all patients, same postoperative rehabilitation program which is indicated below was applied with attention. For the first week walking with a full load, $0^{\circ}-90^{\circ}$ of passive knee extension, bringing the active flexion, quadriceps and hamstring muscle training, heel shift, straight leg rise; between 1-2 weeks, hamstring training in the prone position, asideleg lift, walkingin the water if a swimming pool exist; between 2-3 weeks, terminal extension and hamstring stretch studies with weight; 3-4 weeks, if knee flexion has reached to $90^{\circ}$ the pedals ergonometric work, walking back in the pool (if possible), doing full daily activities; 6-12 weeks, rising at finger tips and starting to closed kinetic chain exercises, preparations for the transition to the sport aiming to increase strength, durability and propsiosepsiyo; In the 3rd month, cycling, running and scissoring in water; in the 4th month, in addition to weight training activities beginning to proprioceptive and on the stairs activities, doing straight running; in 6-8 months beginning to sport-specific movements, ensuring to return to contact sports were taken into account.

Each patient was laid in the supine position, knees at full extension and relaxed position prior to measurement of the circumference of the thigh muscles. Both thigh circumference were measured and recorded from $15 \mathrm{~cm}$ proximal to the upper limit of the patella for measuring. The determined length difference between there constructed knee and the intact knee was taken.

Power measurements of quadriceps and hamstring muscle groups in patients' extremities who underwent operation and who did not inwere done by using Cybex II dynamometer (Humac). During the measurements the maximum torque (peak torque) values at $60 \% \mathrm{sec}, 240 \% \mathrm{sec}$ infrequency, positions of flexion and extension were determined. Measured values between the operated and non-operated extremities have been compared to each other. The application of standard equipment, data collection and heating procedures were performed before the measurement. $\mathrm{Pa}$ tients were told to continue their usual daily activities not to do tiring activities in the day before the test. On the test day before the beginning of measurements patients were carried out a 5-minute warm-up exercises on a stationary bike. Patients' pelvis were stabilized with the help of a belt, thighs were supported with pillows, ankle cuff was placed directly on top of malleolar. Patients' forearms were positioned so that the rotational axis of the forearms were aligned with rotational axis of knees.

During the test the range of motion was adjusted to be $0^{\circ}$ extension and $90^{\circ}$ flexion. In order to get support patients were allowed to hold seats on the sidebar during the test. Before starting 
the recording of the data patients did three times sample repetition at both angle rates. Concentric exercise were carried out for 5 times in maximal flexion and extension to patients after one minute rest. First, $60 \%$ sec speed after a minute rest the test was continued with $240 \%$ sec speed. Patients were encouraged verbally to show maximum effort. Several patients developed thigh pain during measurements and measurements were repeated after the test was terminated.

\section{RESULTS}

Number Cruncher Statistical System (NCSS) 2007 and Power Analysis and Sample Size (PASS) 2008 Statistical Software (Utah, USA) were used for statistical analysis. Besides descriptive statistical methods (mean, standard deviation, median, frequency, rate, minimum, maximum) for qualitative comparison of data Pearson's chi-square test, Fisher-Freeman-Halton test, Fisher's exact test, and Yates Continuity Correction test (Yates adjusted Chisquare) were used for the evaluation of data. Spearman's correlation analysiswas used to assess the relationships between parameters. Significance at $P<0.01$ and $P<0.05$ levels were evaluated.

Table 1. The distribution of descriptive characteristics

\begin{tabular}{lcc} 
& Min-Max & Mean \pm SD \\
\hline Age (yr) & $17-40$ & $28.18 \pm 6.21$ \\
Follow up (month) & $9-42$ & $23.09 \pm 9.08$ \\
\hline
\end{tabular}

Table 3. Distribution of cybex extension and flexion measurement of the patients

\begin{tabular}{llr}
\hline & & \multicolumn{1}{c}{ Mean \pm SD } \\
\hline Cybex Extension 60 & Operated & $192.47 \pm 46.89$ \\
& Normal & $219.27 \pm 32.72$ \\
Cybex Extension 240 & Operated & $94.03 \pm 23.18$ \\
& Normal & $106.41 \pm 17.38$ \\
Cybex Flexion 60 & Operated & $142.63 \pm 29.46$ \\
& Normal & $150.74 \pm 24.22$ \\
Cybex Flexion 240 & Operated & $79.12 \pm 20.85$ \\
& Normal & $87.78 \pm 19.25$ \\
\hline
\end{tabular}

Age identifier values and follow-up periods of patients are shown in Table 1. Thigh circumference of intact extremity and operated extremity of patients are shown in Table 2. Peak torque of the mean extensor and flexor muscle strength and percentage values relative to each other, are shown in Tables 3, 4, and 5.

Statistically significant relationship was observed at the level of 66.0\% percent between the thigh diameter difference and Cybex Extension 60 of patients in negative direction (while the thigh diameter difference increasing the Cybex Extension 60 percent decreasing) (r: $-0,660 ; P=0.001 ; P<0.01$ ).

Statistically significant relationship was observed at the level of $55.0 \%$ percent between the thigh diameter difference and Cybex Extension 240 of patients in negative direction (while the thigh diameter difference increasing the Cybex Extension 240 percent decreasing) (r: $-0,550 ; P=0.005 ; P<0.01)$ (Fig. 1).

Statistically significant relationship was observed at the level of $55.0 \%$ percent between the thigh diameter difference and Cybex Flexion 60 of patients in negative direction (while the thigh diameter difference increasing the Cybex Flexion 60 percent decreasing) (r: $-0,555 ; P=0.002 ; P<0.01)$.

It was observed a relation between the thigh diameter difference

Table 2. Evaluation of patients thigh diameter difference $(n=55)$

\begin{tabular}{llc} 
& & Thigh Diameter Difference \\
& & $\mathrm{n}(\%)$ \\
\hline Total & Min-Max & $0-4$ \\
& Med \pm SD & $1.60 \pm 1.08$ \\
& Medyan & 1
\end{tabular}

Table 4. Evaluation of cybex extension and flexion measurement of the patients

\begin{tabular}{|c|c|c|}
\hline & Transtibial $(\mathrm{n}=27)$ & \multirow{2}{*}{$P$} \\
\hline & Med \pm SD (Median) & \\
\hline Cybex Extension 60 & $88.67 \pm 19.60(91.9)$ & $0.009^{* *}$ \\
\hline Cybex Extension 240 & $88.23 \pm 18.73$ (88.8) & $0.023^{*}$ \\
\hline Cybex Flexion 60 & $93.63 \pm 14.65(96.74)$ & $0.001^{* *}$ \\
\hline Cybex Flexion 240 & $93.25 \pm 31.02(90.03)$ & 0.449 \\
\hline
\end{tabular}

Mann Whitney U Test. ${ }^{*} P<0.05,{ }^{* *} P<0.01$.

Table 5. Distribution of cybex extension and flexion measurement of the patients

\begin{tabular}{lcccccc}
\hline & $r$ & $P$ & $r$ & $P$ & $r$ \\
\hline Cybex Extension 60 Ratio * Thigh difference & -0.823 & $0.001^{* *}$ & -0.391 & 0.089 & -0.66 & $0.001^{* *}$ \\
Cybex Extension 240 Ratio * Thigh difference & -0.366 & 0.112 & -0.426 & 0.061 & -0.55 & $0.005^{* *}$ \\
Cybex Flexion 60 Ratio * Thigh difference & -0.433 & 0.057 & -0.437 & 0.054 & -0.555 & $0.002^{* *}$ \\
Cybex Flexion 240 Ratio Ratio * Thigh difference & -0.126 & 0.597 & -0.375 & 0.103 & -0.281 & 0.079 \\
\hline
\end{tabular}

r. Spearman's Corelation coefficient. ${ }^{*} P<0.05,{ }^{* *} P<0.01$. 


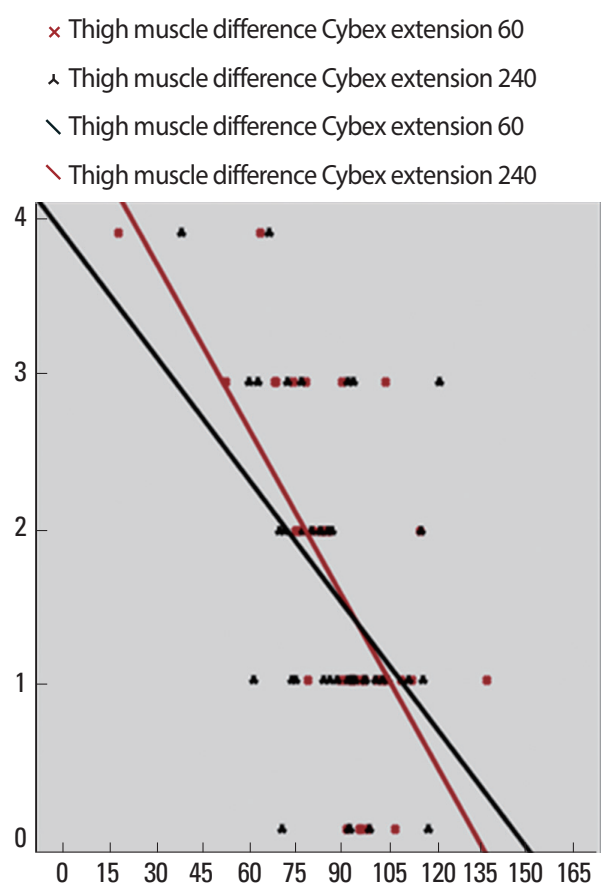

Fig. 1. Cybex extension.

and Cybex Flexion 240 of patients in negative direction (while the thigh diameter difference increasing the Cybex Flexion 240 percent decreasing) at the level of $28.1 \%$ percent and this situation was found to be statistically significant (r: $-0,281 ; P=0.079$; $P>0.05$ ) (Fig. 2).

\section{DISCUSSION}

After our study, in accordance with our findings it is still possible to encounter the thigh atrophy in average 28 months after ACL reconstruction surgery even under physical rehabilitation programs and appropriate follow-up. In line with earlier studies, quadriceps muscle mass is often seen as responsible for the present thigh atrophy, however negative effects of the hamstring muscle group on muscle atrophy which is determined with manual thigh circumference measurements are undeniable. As shown in previous studies, in patients with ACL lesions the development of neurological dysfunction is not available in the knee flexors comparing with quadriceps muscle group.

Comparative studies which was done in patients with ACL lesion and in patients without any knee problems showed that there was no significant difference between the speed of flexion and torque per unit volume of the knee flexor muscle. Despite this, in the same study it was found that isokinetic torque that occurred

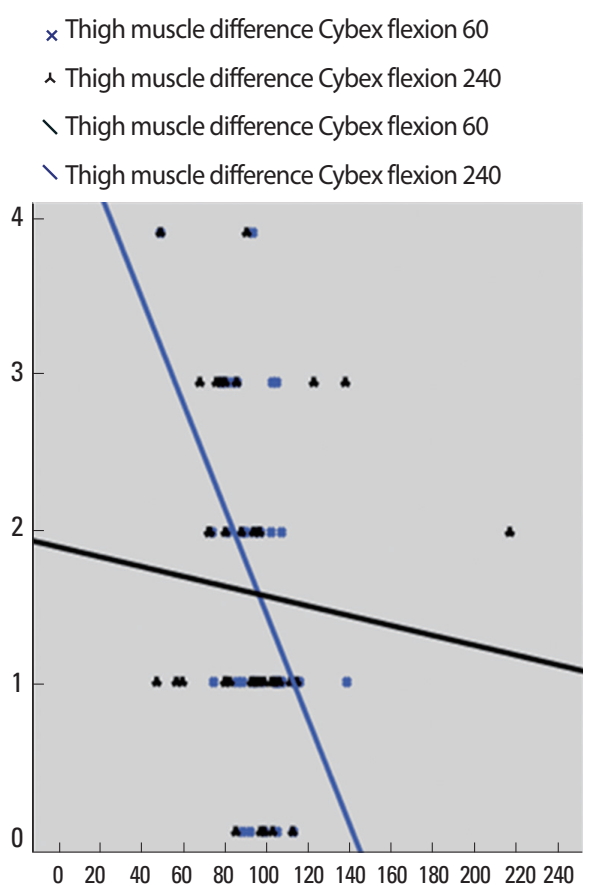

Fig. 2. Cybex flexion.

in the knee with ACL lesions at $60 \%$ sec was less than robust knee (Konishi et al., 2012). In addition, it is shown that the ACL reconstruction with hamstring tendon is not effective on muscle torque power of knee flexors and flexion speed of these muscles by showing that detection of changes in the robust knee is the same. In the light of these data, it might lead to think that ACL lesions does not cause neurological dysfunction in the knee flexors. In another study there was a comparison between patients who were administered to the ACL reconstruction and patients who did not have any knee problem, it has been identified that muscle torque power on per unit volume of the quadriceps was significantly lower in the patients who were administered to reconstruction. In addition, quadriceps weakness is associated with the neurologic dysfunction that is developed after ACL reconstruction has been shown by other researchers (Hart et al., 2010; Rice et al., 2009; Snyder-Mackler et al., 1994; Urbach et al., 1999; 2002). It has been shown that muscle weakness in the quadriceps that is induced by the ACL lesions is a natural result linked to the development of neurological dysfunction.

In our study we have showed the loss of torque power of thigh atrophy on quadriceps at the $60 \% \mathrm{sec}, 240 \% \mathrm{sec}$ frequency even in the patients who have an appropriate rehabilitation program after ACL reconstruction and under going follow-up process. Although, it has been shown in previous studies that ACL lesion de- 
velopment in the thigh did not lead to neurological dysfunction on hamstring; in our measurements, it has been determined statistically that atrophy developed in the thigh is effective on the hamstrings muscle torque power at least as it is on quadriceps, especially $60 \%$ sec frequency. It could not be shown that this weakness is correlated to thigh atrophy as well as quadriceps at $240 \%$ sec flexion frequency.

In addition, treatment of quadriceps weakness developing after ACL injuries is difficult. These difficulties are likely to be related to neurological dysfunction occurred in the quadriceps muscles. Due to different muscle weakness mechanisms which are developing according to the morphological structure and muscle properties, it is inevitable for the clinician to consider these changes in diagnosis and rehabilitation stages. The point that we want to emphasize in our study is; it can't be ignored that muscle weakness mechanisms developing in the thigh circumference vary according to the thigh muscle group and knee flexors play an important role in thigh atrophy when determining an appropriate rehabilitation program after anterior cruciate ligament reconstruction application.

\section{CONFLICT OF INTEREST}

No potential conflict of interest relevant to this article was reported.

\section{REFERENCES}

Eriksson K, Kindblom L, Hamberg P, Larsson H, Wredmark T. The semitendinosus tendon regenerates after resection: a morphologic and MRI analysis in 6 patients after resection for anterior cruciate ligament reconstruction. Acta Orthop Scand 2001;72(4):379-384.

Ferretti A, Conteduca F, Morelli F, Masi V. Regeneration of the semitendinosus tendon after its use in anterior cruciate ligament reconstruction: a histologic study of three cases. Am J Sports Med 2002;30(2):204-207.

Hart JM, Pietrosimone B, Hertel J, Ingersoll CD. Quadriceps activation following knee injuries: a systematic review. J Athl Train 2010;45(1):8797.

Harter RA, Osternig LR, Standifer LW. Isokinetic evaluation of quadriceps and hamstrings symmetry following anterior cruciate ligament reconstruction. Arch Phys Med Rehabil 1990;71(7):465-468.

Hioki S, Fukubayashi T, Ikeda K, Niitsu M, Ochiai N. Effect of harvesting the hamstringstendon for anterior cruciate ligament reconstruction on the morphology and movement of the hamstrings muscle: a novel MRI technique. Knee Surg Sports Traumatol Arthrosc 2003;11(4):223-
227.

Konishi Y, Aihara Y, Sakai M, Ogawa G, Fukubayashi T. Gamma loop dysfunction in the quadriceps femoris of patients who underwent anterior cruciate ligament reconstruction remains bilaterally. Scand J Med Sci Sports 2007a;17:393-399.

Konishi Y, Fukubayashi T, Takeshita D. Possible mechanism of quadriceps femoris weakness in patients with ruptured anterior cruciate ligament. Med Sci Sports Exercise 2002a;34(9):1414-1418.

Konishi Y, Fukubayashi T, Takeshita D. Mechanism of quadriceps femoris muscle weakness in patients with anterior cruciate ligament reconstruction. Scand J Med Sci Sports 2002b;12:371-375.

Konishi Y, Ikeda K, Nishino A, Sunaga M, Aihara Y, Fukubayashi T. Relationship between quadriceps femoris muscle volume and muscle torque after anterior cruciate ligament repair. Scand J Med Sci Sports 2007b;17:656-661.

Konishi Y, Kinugasa R, Oda T, Tsukazaki S, Fukubayashi T. Relationship between muscle volume and muscle torque of the hamstrings after anterior cruciate ligament lesion. Knee Surg Sports Traumatol Arthrosc 2012;20:2270-2274.

Konishi Y, Konishi H, Fukubayashi T. Gamma loop dysfunction in quadriceps on the contralateral side in patients with ruptured ACL. Med Sci Sports Exercise 2003;35(6):897-900.

Lipscomb AB, Johnston RK, Snyder RB, Warburton MJ, Gilbert PP. Evaluation of hamstring strength following use of semitendinosus and gracilis tendons to reconstruct the anterior cruciate ligament. Am J Sports Med 1982;10(6):340-342.

Nakamae A, Deie M, Yasumoto M, Adachi N, Kobayashi K, Yasunaga Y, Ochi M. Three-dimensional computed tomography imaging evidence of regeneration of the semitendinosus tendon harvested for anterior cruciate ligament reconstruction: a comparison with hamstring muscle strength. J Comput Assist Tomogr 2005;29(2):241-245.

Rice DA, McNair PJ. Quadriceps arthrogenic muscle: neural mechanisms and treatment perspectives. Semin Arthritis Rheum 2009;40(3):250266.

Simonian PT, Harrison SD, Cooley VJ, Escabedo EM, Deneka DA, Larson RV. Assessment of morbidity of semitendinosus and gracilis tendon harvest for ACL reconstruction. Am J Knee Surg 1997;10(2):54-59.

Snyder-Mackler L, De Luca PF, Williams PR, Eastlack ME, Bartolozzi AR. 3rd Reflex inhibition of the quadriceps femoris muscle after injury or reconstruction of the anterior cruciate ligament. J Bone Joint Surg Am 1994;76(4):555-560.

Urbach D, Awiszus F. Impaired ability of voluntary quadriceps activation bilaterally interferes with function testing after knee injuries. A twitch interpolation study. Int J Sports Med 2002;23(4):231-236.

Urbach D, Nebelung W, Weiler HT, Awiszus F. Bilateral deficit of volun- 
tary quadriceps muscle activation after unilateral ACL tear. Med Sci Sports Exerc 1999;31(12):1691-1696.

Williams GN, Snyder-Mackler L, Barrance PJ, MJ Axe, Buchanan TS.
Muscle and tendon morphology after reconstruction of the anterior cruciate ligament with autologous semitendinosus-gracilis graft. J Bone Joint Surg Am 2004;86-A(9):1936-1946. 